\title{
QUOTIENTS OF TANGENTIAL $k$-BLOCKS
}

\author{
GEOFFREY WHITTLE
}

(Communicated by Thomas H. Brylawski)

\begin{abstract}
A BSTRACT. A tangential $k$-block over $G F(q)$ is a simple matroid representable over $G F(q)$ with critical exponent $k+1$ for which every proper loopless minor has critical exponent at most $k$. Such matroids are of central importance in the critical problem of Crapo and Rota. In this paper we provide sufficient conditions for a quotient of a tangential $k$-block over $G F(q)$ to be also a tangential $k$-block over $G F(q)$. This enables us to show that there exist rank $r$ supersolvable tangential $k$-blocks over $G F(q)$ exactly when $q^{k} \geq r \geq k+1$.
\end{abstract}

1. Introduction. A tangential $k$-block over $G F(q)$ is a simple matroid representable over $G F(q)$ with critical exponent $k+1$ for which every loopless minor has critical exponent at most $k$. If $M$ and $M^{\prime}$ are matroids sharing a common ground set then $M^{\prime}$ is a quotient of $M$ if every flat of $M^{\prime}$ is also a flat of $M$. In this paper we relate the theory of quotients of matroids to tangential $k$-blocks. This enables us to answer a number of questions about tangential $k$-blocks and to considerably extend the class of known tangential $k$-blocks.

The main theorem (3.1) of this paper provides sufficient conditions for the simple matroid associated with a quotient of a tangential $k$-block over $G F(q)$ to be also a tangential $k$-block over $G F(q)$. This enables us to show that there exist rank $r$ tangential $k$-blocks over $G F(q)$ whenever $q^{k} \geq r \geq k+1$ and also that there exist supersolvable rank $r$ tangential $k$-blocks over $G F(q)$ exactly when $q^{k} \geq r \geq k+1$. As a consequence a conjecture of Welsh $[\mathbf{7}, \mathbf{8}]$, namely that the only tangential 3-blocks over $G F(2)$ are $M\left(K_{9}\right)$ and $P G(3,2)$, is shown to be false. Example 3.6 gives matrix representations of tangential 3-blocks over $G F(2)$ of ranks 4, 5, 6, 7 and 8.

Results on the characteristic polynomial of tangential $k$-blocks obtained via the process of taking quotients of other tangential $k$-blocks are also included.

2. Definitions and preliminary results. We assume that the reader is familiar with the basic concepts of matroid theory, particularly that of the characteristic polynomial $P(M ; \lambda)$ of a matroid and the critical exponent $c(M ; q)$ of a matroid representable over $G F(q)$. Free use will be made of results in Welsh [6, Chapter 16] which provides a good introduction to these topics. The terminology used here will in general follow Welsh [6]. The set of elements of a matroid $M$ will be denoted by $E(M)$ and if $S=E(M)$ we say that $M$ is a matroid on $S$. If $T \subseteq E(M)$, the restriction of $M$ to $E(M) \backslash T$ will be denoted by $M \mid(E(M) \backslash T)$ or by $M \backslash T$ and the contraction of $M$ to $E(M) \backslash T$ will be denoted by $M \cdot(E(M) \backslash T)$ or by $M / T$ in either case according to convenience. The closure and rank of $T$ in $M$ will be denoted by $\mathrm{cl}_{M}(T)$ and $r_{M}(T)$ respectively or if no danger of ambiguity exists by $\operatorname{cl}(T)$ and

Received by the editors May 14, 1986.

1980 Mathematics Subject (Classification (1985 Revision). Primary 05B35. 
$r(T)$ respectively. The simple matroid associated with $M$ will be denoted by $\bar{M}$. The complete graph with $n$ vertices will be denoted by $K_{n}$. The cycle matroid of a graph $G$ will be denoted by $M(G)$.

Tangential $k$-blocks over $G F(q)$. A matroid $M$ is a tangential $k$-block over $G F(q)$ if the following conditions hold:

(a) $M$ is simple and representable over $G F(q)$,

(b) $P\left(M ; q^{k}\right)=0$,

(c) $P\left(M / F ; q^{k}\right)>0$ whenever $F$ is a proper nonempty flat of $M$.

Note that condition (c) above is equivalent to the apparently stronger

(c') $P\left(M^{\prime} ; q^{k}\right)>0$ whenever $M^{\prime}$ is a proper loopless minor of $M$.

In the language of critical exponents a simple matroid representable over $G F(q)$ is a tangential $k$-block over $G F(q)$ if $C(M ; q)>k$ and whenever $F$ is a proper nonempty flat of $M, c(M / F ; q) \leq k$.

A matroid $M$ is a tangential $k$-block if there exists a prime power $q$ such that $M$ is a tangential $k$-block over $G F(q)$.

It is well known that for any prime power $q$, both $M\left(K_{q^{k}+1}\right)$ and $P G(k, q)$ are tangential $k$-blocks over $G F(q)$ and that

$$
P\left(M\left(K_{q^{k}+1}\right) ; \lambda\right)=(\lambda-1)(\lambda-2) \cdots\left(\lambda-q^{k}\right)
$$

and that

$$
P(P G(k, q) ; \lambda)=(\lambda-1)(\lambda-q) \cdots\left(\lambda-q^{k}\right) .
$$

Modular flats. A flat $F$ of a matroid $M$ is modular if $r(F)+r\left(F^{\prime}\right)=r\left(F \cup F^{\prime}\right)+$ $r\left(F \cap F^{\prime}\right)$ for every flat $F^{\prime}$ of $M$.

PROPOSITION 2.1. If $F$ is a rank $k$ flat of a matroid $M$ and there exists $X \subset F$ such that $F \backslash X$ is a rank $k$ modular flat of $M \backslash X$ then $F$ is a modular flat of $M$.

ProOF. In [1] Brylawski shows that a flat $F$ is modular if and only if for all flats $F^{\prime}$ of $M$ with $r\left(F \cap F^{\prime}\right)=0, r(F)+r\left(F^{\prime}\right)=r\left(F \cup F^{\prime}\right)$. The result is a routine consequence of this observation.

The following proposition is essentially a combination of $[\mathbf{1}$, Theorem 3.3 .8 and Corollary 3.9] although Brylawski's language is lattice theoretic. For a matroid theoretic proof see [10, Corollary 3.1.9].

PROPOSITION 2.2. If $F$ is a modular flat of the matroid $M$ and $A \subseteq E(M)$ then $\mathrm{cl}_{M / A}(F \backslash A)$ is a modular flat of $M / A$. Furthermore, if $A$ is a flat of $M$ then $\overline{(M / A) \mid \mathrm{cl}_{M / A}(F \backslash A)} \cong \overline{(M /(A \cap F)) \mid(F \backslash A)}$.

The statement that $\overline{(M / A) \mid \mathrm{cl}_{M / A}(F \backslash A)} \cong \overline{(M /(A \cap F)) \mid(F \backslash A)}$ needs careful reading. It tells us that the simple matroid associated with the restriction of $M / A$ to the flat $\mathrm{cl}_{M / A}(F \backslash A)$ of $M / A$ is isomorphic to the simple matroid associated with the restriction of $M /(A \cap F)$ to the flat $F \backslash A$ of $M /(A \cap F)$. Note that $F \backslash A$ is a flat of $M /(A \cap F)$ since $(F \backslash A) \cup(A \cap F)=F$; a flat of $M$.

The complete Brown truncation. Let $M$ be a rank $r$ matroid and $F$ be a rank $k$ flat of $M$ where $k \geq 1$, then as defined in [2] the complete Brown truncation of $M$ by $F$, denoted $T_{F}(M)$, is the matroid whose bases are subsets of $E(M)$ of the form $B$ or $B^{\prime} \cup x, x \in F$ ( $x$ not a loop) where $B$ and $B^{\prime}$ are independent subsets (in $M$ ) of $E(M) \backslash F$ with $|B|=r-k+1,\left|B^{\prime}\right|=r-k$ and $r(B \cup F)=r\left(B^{\prime} \cup F\right)=r$. If $S \subseteq E(M)$ is not a flat then $T_{S}(M)=T_{\mathrm{cl}_{M}(S)}(M)$. 
Most applications of $T_{F}(M)$ occur when $F$ is modular. In this case the bases of $T_{F}(M)$ can be characterised more simply.

PROPOSITION 2.3. If $F$ is a modular flat of the matroid $M$ with $r(F) \geq 1$ then the bases of $T_{F}(M)$ are the subsets $B$, independent in $M$ with $|B|=r-k+1$ and $r(B \cup F)=r$.

Proof. This is [9, Proposition 2.5].

The stimulus for the study of modular flats and the complete Brown truncation in the context of the critical problem comes from the following fundamental result.

PROPOSITION 2.4 (BRYLAWSKI [1, COROLlARY 7.4]). If $F$ is a modular flat of the matroid $M$ then

$$
P(M ; \lambda)=P\left(T_{F}(M) ; \lambda\right) P(M \mid F ; \lambda) /(\lambda-1) .
$$

Supersolvable matroids. Let $M$ be a rank $r$ matroid, then $M$ is said to be supersolvable if there exists a set $\left\{F_{0}, F_{1}, F_{2}, \ldots, F_{r}\right\}$ of modular flats of $M$ with $r\left(F_{i}\right)=i$ for $0 \leq i \leq r$ and for $1 \leq i \leq r, F_{i} \supseteq F_{i-1}$. We say that $\left\{F_{0}, F_{1}, \ldots, F_{r}\right\}$ is a saturated chain of modular flats.

Supersolvable matroids were introduced by Stanley $[\mathbf{4}, \mathbf{5}]$ where he proves

PROPOSITION 2.5. If the simple matroid $M$ is supersolvable with saturated chain of modular flats $\left\{F_{0}, F_{1}, \ldots, F_{r}\right\}$ then

$$
P(M ; \lambda)=\prod_{i=1}^{r}\left(\lambda-\left|F_{i} \backslash F_{i-1}\right|\right) .
$$

Quotients of matroids. Let $M$ and $M^{\prime}$ be matroids with $E(M)=E\left(M^{\prime}\right)$. Then $M^{\prime}$ is a quotient of $M$ if every flat of $M^{\prime}$ is also a flat of $M$. If $r(M)-r\left(M^{\prime}\right)=1$ then $M^{\prime}$ is an elementary quotient of $M$. The theory of quotients of matroids belongs to the theory of strong maps and single element extensions of matroids developed by Crapo and Rota [3, Chapter 10]. Welsh [6, Chapter 17] gives an excellent survey of the theory. The results summarised in Proposition 2.6 below are either contained in, or immediately derivable from, results in Welsh [6, Chapter 17].

Let $M^{\prime \prime}$ be a matroid and $M=M^{\prime \prime} \backslash P$, then $M^{\prime \prime}$ is an extension of $M$ by $P$. If $P$ is independent in $M^{\prime \prime}$ then $M^{\prime \prime}$ is an independent extension of $M$ by $P$.

Proposition 2.6. Let $M$ and $M^{\prime}$ be matroids with $E(M)=E\left(M^{\prime}\right)=E$.

(i) $M^{\prime}$ is an elementary quotient of $M$ if and only if there exists a matroid $M^{\prime \prime}$ having ground set $E \cup p$ with $p$ independent in $M^{\prime \prime}$ and $M^{\prime \prime} \mid E=M$ (that is, an independent single point extension of $M$ ) with the property that $M^{\prime \prime} / p=M^{\prime}$. The matroid $M^{\prime \prime}$ is, up to isomorphism, unique.

(ii) Assume $r(M)-r\left(M^{\prime}\right)=k$ and let $P$ be disjoint from $E$ with $|P|=k . M^{\prime}$ is a quotient of $M$ if and only if there exists an independent extension of $M$ by $P$, say $M^{\prime \prime}$, with $M^{\prime \prime} / P=M^{\prime}$. $M^{\prime \prime}$ is not necessarily unique.

(iii) Let $M^{\prime}$ be a quotient of $M$ with $r(M)-r\left(M^{\prime}\right)=k$ and let $M^{\prime \prime}$ be an extension of $M$ by $P$ (not necessarily independent) with $M^{\prime \prime} / P=M^{\prime}$. A flat $F$ of $M^{\prime}$ has $r_{M}(F)-r_{M^{\prime}}(F)=k$ if and only if $P \subseteq \operatorname{cl}_{M^{\prime \prime}}(F)$. In fact, for any flat $F$ of $M^{\prime}$, $r_{M}(F)-r_{M^{\prime}}(F)=r_{M^{\prime \prime}}\left(P \cap \mathrm{cl}_{M^{\prime \prime}}(F)\right)$.

We are interested in modular flats. For quotients we have the following proposition. 
PROPOSITION 2.7. Let $M^{\prime}$ be a quotient of the matroid $M$ and $F$ be a flat of $M^{\prime}$ which is modular in $M$. If $r_{M}(F)-r_{M^{\prime}}(F)=r(M)-r\left(M^{\prime}\right)$, then $F$ is modular in $M^{\prime}$.

PrOOF. By Proposition 2.6(ii), there exists an independent extension $M^{\prime \prime}$ of $M$ by a set $P$ with the property that $M^{\prime \prime} / P=M^{\prime}$. By Proposition 2.6(iii), $P \subseteq$ $\operatorname{cl}_{M^{\prime \prime}}(F)$, hence by Proposition $2.1, F \cup P$ is a modular flat of $M^{\prime \prime}$. Therefore by Proposition $2.2, F$ is a modular flat of $M^{\prime}=M^{\prime \prime} / P$.

Proposition 2.8 below connects quotients, modular flats and the complete Brown truncation.

PROPOSITION 2.8. Let $M^{\prime}$ be a loopless quotient of the simple matroid $M$ and $F$ be a flat of $M^{\prime}$ which is modular in $M$. If $r_{M}(F)-r_{M^{\prime}}(F)=r(M)-r\left(M^{\prime}\right)$, then $T_{F}(M)=T_{F}\left(M^{\prime}\right)$.

ProOF. By Proposition 2.6(ii), there exists an independent extension $M^{\prime \prime}$ of $M$ by a set $P$ with the property that $M^{\prime \prime} / P=M^{\prime}$. Assume $M$ has rank $r$ and $M \mid F$ has rank $k$. Since $M^{\prime}$ is loopless $|P|<k$, say $|P|=j$. Then $r\left(M^{\prime}\right)=r-j$ and $r\left(M^{\prime} \mid F\right)=k-j$ and therefore $r\left(T_{F}\left(M^{\prime}\right)\right)=r\left(T_{F}(M)\right)=r-k+1$. Now $F$ is modular in $M$ and by Proposition $2.7, F$ is modular in $M^{\prime}$. Therefore by Proposition 2.3, bases of $T_{F}(M)$ (respectively $T_{F}\left(M^{\prime}\right)$ ) are subsets $B$ of $E(M)$, independent in $M$ (respectively $M^{\prime}$ ) with $|B|=r-k+1$ and $r_{M}(B \cup F)=r$ (respectively $\left.r_{M^{\prime}}(B \cup F)=r-j\right)$.

Assume that $B$ is a basis of $T_{F}(M)$, then since $F$ is modular in $M$, $r\left(\operatorname{cl}_{M}(B) \cap F\right)=1$. Say $f=\operatorname{cl}_{M}(B) \cap F$ (well defined since $M$ is simple). Now $F \cup P$ is a rank $k$ modular flat of $M^{\prime \prime}$ so $r\left(\operatorname{cl}_{M^{\prime \prime}}(B) \cap F\right)=1$. Also $\operatorname{cl}_{M^{\prime \prime}}(f)=f$ (otherwise $M^{\prime}$ is not loopless) so $\operatorname{cl}_{M^{\prime \prime}}(B) \cap f=f$. Since $M^{\prime}$ is loopless, $\operatorname{cl}_{M^{\prime \prime}}(P)=$ $P$ so $P \cup f$ is independent in $M^{\prime \prime}$. Extend $P \cup f$ to a basis $B^{\prime}$ of $M^{\prime \prime} \mid(F \cup P)$. Since $\operatorname{cl}_{M^{\prime \prime}}\left(B^{\prime}\right)=F \cup P$ we have,

$$
r_{M^{\prime \prime}}\left(B^{\prime} \cup B\right)=r_{M^{\prime \prime}}\left(B^{\prime}\right)+r_{M^{\prime \prime}}(B)-r_{M^{\prime \prime}}\left(F \cap \operatorname{cl}_{M^{\prime \prime}}(B)\right)=r .
$$

If $f \in B$ then $\left|B^{\prime} \cup B\right|=r$ and $B^{\prime} \cup B$ is therefore independent in $M^{\prime \prime}$ and in particular $P \cup B$ is independent in $M^{\prime \prime}$. If $f \notin B$ then $\left|B^{\prime} \cup B\right|=r+1$. Now $f$ is not a coloop of $M^{\prime \prime} \mid\left(B^{\prime} \cup B\right)\left(f \in \mathrm{cl}_{M^{\prime \prime} \mid\left(B^{\prime} \cup B\right)}(B)\right)$, hence $r_{M^{\prime \prime}}\left(\left(B^{\prime} \backslash f\right) \cup B\right)=r$. That is $\left(B^{\prime} \backslash f\right) \cup B$ is independent in $M^{\prime \prime}$ and in particular $P \cup B$ is independent in $M^{\prime \prime}$. Since in either case $P \cup B$ is independent in $M^{\prime \prime}, B$ is independent in $M^{\prime \prime} / P=M^{\prime}$. Also

$$
r_{M^{\prime}}(B \cup F)=r_{M^{\prime \prime}}(B \cup F \cup P)-r_{M^{\prime \prime}}(P)=r-j .
$$

Hence $B$ is a basis of $T_{F}\left(M^{\prime}\right)$.

On the other hand assume that $B$ is a basis of $T_{F}\left(M^{\prime}\right)$. Certainly $B$ is independent in $M$ and since $r_{M^{\prime}}(B \cup F)=r-j$, then $r_{M^{\prime \prime}}(B \cup F \cup P)=r$. But $P \subseteq \operatorname{cl}_{M^{\prime \prime}}(F)$ so $r_{M^{\prime \prime}}(B \cup F)=r$ and therefore $r_{M}(B \cup F)=r$. That is, $B$ is a basis of $T_{F}(M)$ and the proposition is proved.

3. Construction of tangential $k$-blocks via quotients. The following theorem is our main result. It provides sufficient conditions for the simple matroid associated with a loopless quotient of a tangential $k$-block over $G F(q)$ to be also a tangential $k$-block over $G F(q)$. 
THEOREM 3.1. Let $M$ be a tangential $k$-block over $G F(q)$ and $M^{\prime}$ be a loopless quotient of $M$ which is representable over $G F(q)$ with $r(M)-r\left(M^{\prime}\right)=m$. Assume there exists a proper nonempty flat $F$ of $M^{\prime}$ with the following properties:

(i) $F$ is a modular flat of $M$,

(ii) $r_{M}(F)-r_{M^{\prime}}(F)=m$,

(iii) for every proper flat $X$ of $M^{\prime} \mid F, P\left(\left(M^{\prime} \mid F\right) / X ; q^{k}\right)>0$.

Then $\overline{M^{\prime}}$ is a tangential $k$-block over $G F(q)$.

ProOF. Since $F$ is modular in $M$, we see by Proposition 2.7 that $F$ is modular in $M^{\prime}$ and therefore

$$
P\left(M^{\prime} ; q^{k}\right)=P\left(M \mid F ; q^{k}\right) P\left(T_{F}\left(M^{\prime}\right) ; q^{k}\right) /\left(q^{k}-1\right) .
$$

Also, since $M$ is a tangential $k$-block over $G F(q)$,

$$
P\left(M ; q^{k}\right)=P\left(M \mid F ; q^{k}\right) P\left(T_{F}(M) ; q^{k}\right) /\left(q^{k}-1\right)=0 .
$$

But $F$ is a proper flat of $M$ so $P\left(M \mid F ; q^{k}\right)>0$, hence $P\left(T_{F}(M) ; q^{k}\right)=0$. By Proposition $2.8, T_{F}(M)=T_{F}\left(M^{\prime}\right)$ so $P\left(T_{F}\left(M^{\prime}\right) ; q^{k}\right)=0$ and therefore $P\left(M^{\prime} ; q^{k}\right)=0$. But $M^{\prime}$ is loopless so $P\left(\overline{M^{\prime}} ; q^{k}\right)=P\left(M^{\prime} ; q^{k}\right)=0$.

We now show that if $A$ is a proper nonempty flat of $M^{\prime}$, then $P\left(M^{\prime} / A ; q^{k}\right)>0$. By Proposition 2.6(ii) there exists an independent extension $M^{\prime \prime}$ of $M$ by a set $P$ with the property that $M^{\prime \prime} / P=M^{\prime}$. Let $A$ be a proper nonempty flat of $M^{\prime}$ and consider $M^{\prime} / A$.

Assume that $F \subseteq A$. By Proposition 2.6(iii), $P \subseteq \operatorname{cl}_{M^{\prime \prime}}(F)$, hence $P \subseteq \operatorname{cl}_{M^{\prime \prime}}(A)$ so $P$ is a set of loops of $M^{\prime \prime} / A$ and therefore $M^{\prime \prime} / A \backslash P=M^{\prime \prime} / A / P$. Now

$$
M^{\prime} / A=M^{\prime \prime} / P / A=M^{\prime \prime} / A / P=M^{\prime \prime} / A \backslash P=M^{\prime \prime} \backslash P / A=M / A .
$$

But $M^{\prime}$ is a quotient of $M$ and $A$ is a proper nonempty flat of $M^{\prime}$ so $A$ is a proper nonempty flat of $M$. Therefore $P\left(M / A ; q^{k}\right)>0$ and hence $P\left(M^{\prime} / A ; q^{k}\right)>0$.

Assume that $F \backslash A \neq \varnothing$. Since $F$ is a modular flat of $M^{\prime}$ we see by Proposition 2.2 that $\mathrm{cl}_{M^{\prime} / A}(F \backslash A)$ is a modular flat of $M^{\prime} / A$. Let $F^{\prime}=\operatorname{cl}_{M^{\prime} / A}(F \backslash A)$, then since $F \neq \varnothing$ and $F^{\prime}$ is modular in $M^{\prime} / A$, we have

$$
P\left(M^{\prime} / A ; q^{k}\right)=P\left(\left(M^{\prime} / A\right) \mid F^{\prime} ; q^{k}\right) P\left(T_{F^{\prime}}\left(M^{\prime} / A\right) ; q^{k}\right) /\left(q^{k}-1\right) .
$$

We now show that $T_{F^{\prime}}\left(M^{\prime} / A\right)=T_{F^{\prime}}(M / A)$ and therefore that $P\left(T_{F^{\prime}}\left(M^{\prime} / A\right) ; q^{k}\right)$ $>0$. Consider $F^{\prime}=\operatorname{cl}_{M^{\prime} / A}(F \backslash A)$. We have

$$
\operatorname{cl}_{M^{\prime \prime} / A}(F \backslash A)=\operatorname{cl}_{M^{\prime \prime}}((F \backslash A) \cup A) \backslash A \supseteq \operatorname{cl}_{M^{\prime \prime}}(F) \backslash A \supseteq P .
$$

Now $x \in \operatorname{cl}_{M^{\prime} / A}(F \backslash A)$ if and only if $x \in \operatorname{cl}_{M^{\prime \prime} / A}((F \backslash A) \cup P) \backslash P$. But $P \subseteq$ $\operatorname{cl}_{M^{\prime \prime} / A}(F \backslash A)$ so $\mathrm{cl}_{M^{\prime \prime} / A}((F \backslash A) \cup P)=\operatorname{cl}_{M^{\prime \prime} / A}(F \backslash A)$. That is, $x \in \operatorname{cl}_{M^{\prime} / A}(F \backslash A)$ if and only if $x \in \mathrm{cl}_{M^{\prime \prime} / A}(F \backslash A) \backslash P$. But $\mathrm{cl}_{M^{\prime \prime} / A}(F \backslash A) \backslash P=\mathrm{cl}_{M / A}(F \backslash A)$. Hence

$$
\operatorname{cl}_{M^{\prime} / A}(F \backslash A)=\operatorname{cl}_{M / A}(F \backslash A)=F^{\prime} .
$$

(Note that it is not sufficient to show that $F^{\prime}$ is a flat of $M / A$; we need the stronger statement that $F^{\prime}=\operatorname{cl}_{M / A}(F \backslash A)$.) Now $M^{\prime} / A=\left(M^{\prime \prime} / P\right) / A=\left(M^{\prime \prime} / A\right) / P$. Therefore $M^{\prime} / A$ is a quotient of $M / A=\left(M^{\prime \prime} / A\right) \backslash P$. Since $P \subseteq \operatorname{cl}_{M^{\prime \prime} / A}(F \backslash A)$ we see by Proposition 2.6(iii) that $r_{M / A}\left(F^{\prime}\right)-r_{M^{\prime} / A}\left(F^{\prime}\right)=r(M / A)-r\left(M^{\prime} / A\right)$. 
Now $F$ is modular in $M$ so by Proposition $2.2, F^{\prime}=\operatorname{cl}_{M / A}(F \backslash A)$ is modular in $M / A$. Hence by Proposition $2.8, T_{F^{\prime}}(M / A)=T_{F^{\prime}}\left(M^{\prime} / A\right)$. But

$$
P\left(M / A ; q^{k}\right)=P\left((M / A) \mid F^{\prime} ; q^{k}\right) P\left(T_{F^{\prime}}(M / A) ; q^{k}\right) /\left(q^{k}-1\right)
$$

and $P\left(M / A ; q^{k}\right)>0$ so $P\left(T_{F^{\prime}}(M / A) ; q^{k}\right)>0$. Therefore $P\left(T_{F^{\prime}}\left(M^{\prime} / A\right) ; q^{k}\right)>0$.

We now show that $P\left(\left(M^{\prime} / A\right) \mid F^{\prime} ; q^{k}\right)>0$. By Proposition $2.2, \overline{\left(M^{\prime} / A\right) \mid F^{\prime}} \cong$ $\overline{\left(M^{\prime} \mid F\right) /(A \cap F)}$. Now $A \cap F$ is a flat of $M^{\prime} \mid F$ and $A \cap F \neq F$ so by assumption (iii) in the statement of the theorem, $P\left(\left(M^{\prime} \mid F\right) /(A \cap F) ; q^{k}\right)>0$ and therefore (since $\left(M^{\prime} / A\right) \mid F^{\prime}$ is loopless $), P\left(\left(M^{\prime} / A\right) \mid F^{\prime} ; q^{k}\right)>0$.

Summarising we have,

$$
P\left(\left(M^{\prime} / A\right) \mid F^{\prime} ; q^{k}\right)>0, \quad P\left(T_{F^{\prime}}\left(M^{\prime} / A\right) ; q^{k}\right)>0
$$

and

$$
P\left(M^{\prime} / A ; q^{k}\right)=P\left(\left(M^{\prime} / A\right) \mid F^{\prime} ; q^{k}\right) P\left(T_{F^{\prime}}\left(M^{\prime} / A\right) ; q^{k}\right) /\left(q^{k}-1\right),
$$

so $P\left(M^{\prime} / A ; q^{k}\right)>0$.

Since minors obtained by contracting out flats in $M^{\prime}$ differ from minors obtained by contracting out flats in $\overline{M^{\prime}}$ only in respect of parallel elements we conclude that $P\left(\overline{M^{\prime}} / A ; q^{k}\right)>0$ whenever $A$ is a proper nonempty flat of $\overline{M^{\prime}}$ and therefore $\overline{M^{\prime}}$ is a tangential $k$-block over $G F(q)$.

In [9] it is shown that if $M$ is a tangential $k$-block over $G F(q)$ and $F$ is a modular flat of $M$, then whenever $T_{F}(M)$ is representable over $G F(q), \overline{T_{F}(M)}$ is a tangential $k$-block over $G F(q)$. We note here that this result is a routine corollary of Theorem 3.1.

THEOREM 3.2. If $M$ is a supersolvable rank $r$ tangential $k$-block over $G F(q)$ with $r>k+1$, then there exists an elementary quotient $M^{\prime}$ of $M$ with the property that $\overline{M^{\prime}}$ is a supersolvable tangential $k$-block over $G F(q)$.

Proof. Assume that $M=P G(r-1, q) \mid E$ and $M$ has a saturated chain of modular flats $\left\{\varnothing=F_{0}, F_{1}, \ldots, F_{r-1}, F_{r}=E\right\}$. Since $r(M)>k+1, M \not P G(k, q)$. Let

$$
S=\left\{i ; i \in\{2, \ldots, r\} \text { and } M \mid F_{i} \not P G(i-1, q)\right\}
$$

and let $m$ be the least element of $S$. Now $m \leq k+1$ (otherwise $M$ has $P G(k, q)$ as a minor) and $r>k+1$ so $F_{m}$ is a proper flat of $M$. Since $M \mid F \not$ $P G(m-1, q), \operatorname{cl}_{P G(r-1, q)}\left(F_{m}\right) \backslash F_{m} \neq \varnothing$. Let $x \in \operatorname{cl}_{P G(r-1, q)}\left(F_{m}\right) \backslash F_{m}$, let $M^{\prime \prime}=$ $P G(r-1, q) \mid(E \cup x)$ and let $M^{\prime}=M^{\prime \prime} / x . M^{\prime}$ is a loopless elementary quotient of $M$ and since $x \in \operatorname{cl}_{M^{\prime \prime}}\left(F_{m}\right), r_{M}\left(F_{m}\right)-r_{M^{\prime}}\left(F_{m}\right)=1$. Now $r_{M^{\prime}}\left(F_{m}\right) \leq k$ so for every proper flat $F^{\prime}$ of $M^{\prime} \mid F_{m}, P\left(\left(M^{\prime} \mid F_{m}\right) / F^{\prime} ; q^{k}\right)>0$. Therefore, since $F_{m}$ is a proper modular flat of $M$ we have established by Theorem 3.1 that $\overline{M^{\prime}}$ is a tangential $k$-block over $G F(q)$. It remains to show that $\overline{M^{\prime}}$ is supersolvable.

Since $F_{m-1} \cong P G(m-2, q)$ and $x$ is a coloop of $M^{\prime \prime}\left|\left(F_{m-1} \cup x\right), M^{\prime}\right| F_{m-1} \cong$ $P G(m-2, q)$ and therefore $\overline{M^{\prime} \mid F_{m}} \cong P G(m-2, q)$. Therefore any subflat (in $M^{\prime}$ ) of $F_{m}$ is modular in $M^{\prime}$. Now $F_{m} \cup x$ is a modular flat of $M^{\prime \prime}$, hence by Proposition 2.2, $F_{i}$ is a modular flat of $M^{\prime}$ for $m \leq i \leq r$. Given the above one easily constructs a saturated chain of modular flats of $M^{\prime}$ thus demonstrating that $M^{\prime}$ (and therefore $\overline{M^{\prime}}$ ) is supersolvable. 
Since for every prime power $q, M\left(K_{q^{k}+1}\right)$ is a tangential $k$-block over $G F(q)$, we have as an immediate corollary to Theorem 3.2 ,

COROLLARY 3.3. There exists a rank $r$ supersolvable tangential $k$-block over $G F(q)$ whenever $q^{k} \geq r \geq k+1$.

In [9] it is shown that no tangential $k$-block over $G F(q)$ with rank greater than $q^{k}$ has a modular hyperplane. We therefore have the following strengthening of Corollary 3.3.

COROLlARY 3.4. There exists a rank $r$ supersolvable tangential $k$-block over $G F(q)$ if and only if $q^{k} \geq r \geq k+1$.

The above corollaries strengthen results obtained in [9] where it is shown that there exist supersolvable tangential $k$-blocks over $G F(q)$ of all ranks $r$ where $q^{k} \geq$ $r \geq q^{k}-q+2$.

In $[7,8]$ Welsh asks if the only tangential 3-blocks over $G F(2)$ are $M\left(K_{9}\right)$ and $P G(3,2)$. We see now that there are at least 3 others occupying ranks 7,6 and 5 , these being the intermediate ranks between $r\left(M\left(K_{9}\right)\right)$ and $r(P G(3,2))$. Welsh also asks if there is no nongraphic tangential 3-block other than $P G(3,2)$. Again the answer is negative. We explicitly give 3 nongraphic tangential 3-blocks other than $P G(3,2)$ in Example 3.7.

One of the reasons for studying tangential $k$-blocks is the hope that, for a given field and a fixed $k$, there would be relatively few of them. In the light of Corollary 3.3, this hope would seem to be dashed. But supersolvable tangential $k$-blocks form a tractable class and the existence of a number of them over a given field is not of great concern. One could instead ask whether, over a given field for a fixed value of $k$, there exist relatively few nonsupersolvable tangential $k$-blocks (or at least relatively few not containing modular hyperplanes). Given the persistence of Tutte's tangential 2-block conjecture this is likely to be an extremely difficult question to settle (except possibly in the negative).

We now turn our attention to characteristic polynomials. In Theorem 3.5 below we identify the characteristic polynomials of tangential $k$-blocks obtained by starting from $M\left(K_{q^{k}+1}\right)$ and repeatedly using the technique employed in proving Theorem 3.2.

THEOREM 3.5. Let $q$ be a prime power, $k$ a positive integer and $r$ a positive integer with $q^{k} \geq r \geq k+1$. Let $s$ be the greatest integer with $q^{s}-s<q^{k}-r+2$ and let $j=q^{k}-r+s+2$. Then there exists a rank $r$ supersolvable tangential $k$-block $M$ over $G F(q)$ with characteristic polynomial

$$
P(M ; \lambda)=(\lambda-1)(\lambda-q) \cdots\left(\lambda-q^{s}\right)(\lambda-j)(\lambda-(j+1)) \cdots\left(\lambda-q^{k}\right) .
$$

Furthermore the ordering of the above factorisation of $P(M ; \lambda)$ is induced by a saturated chain of modular flats of $M$.

PROOF. If $r=q^{k}$ then $q=2, s=1$ and $j=3$ or $q>2, s=0$ and $j=2$. In either case the polynomial obtained is $(\lambda-1)(\lambda-2) \cdots\left(\lambda-q^{k}\right)$ which is the characteristic polynomial of $M\left(K_{q^{k}+1}\right)$. But $M\left(K_{q^{k}+1}\right)$ is supersolvable tangential $k$-block over $G F(q)$ and the above factorisation of $P\left(M\left(K_{q^{k}+1}\right) ; \lambda\right)$ is certainly induced by a saturated chain of modular flats of $M\left(K_{q^{k}+1}\right)$ so the result holds for $r=q^{k}$. 
Assume that the result holds for $r=m$ where $q^{k} \geq m>k+1$ and let $M$ be a rank $m$ supersolvable tangential $k$-block with characteristic polynomial

$$
P(M ; \lambda)=(\lambda-1)(\lambda-q) \cdots\left(\lambda-q^{s}\right)(\lambda-j)(\lambda-(j+1)) \cdots\left(\lambda-q^{k}\right)
$$

where $s$ and $j$ are as defined in the statement of the theorem and the above factorisation of $P(M ; \lambda)$ is induced by a saturated chain of modular flats of $M$. Say $\left\{\phi=F_{0}, F_{1}, \ldots, F_{m-1}, F_{m}\right\}$ is such a saturated chain of modular flats. Assume that $M=P G(m-1, q) \mid E$ for some set $E$ of points of $P G(m-1, q)$. Now

$$
P\left(M \mid F_{s+1} ; \lambda\right)=(\lambda-1)(\lambda-q) \cdots\left(\lambda-q^{s}\right)
$$

and since $M$ is a subgeometry of $P G(m-1, q)$ it is routine to show that $M \mid F_{s+1} \cong$ $P G(s, q)$. Since $m>k+1, s<k$ and $F_{s+1}$ is a proper flat of $M$. Consider $M \mid F_{s+2}$. It is routine to show that $q^{s}<j<q^{s+1}$ and that $M \mid F_{s+2} \cong P G(s+1, q)$ if and only if $j=q^{s+1}$. Therefore $M \mid F_{s+2} \not \nexists P G(s+1, q)$ and there exists $x \in$ $\operatorname{cl}_{P G(m-1, q)}\left(F_{s+2}\right) \backslash F_{s+2}$. Let $M^{\prime \prime}=P G(m-1, q) \mid E \cup x$. One readily sees that $P\left(M^{\prime \prime} ; \lambda\right)=(\lambda-1)(\lambda-q) \cdots\left(\lambda-q^{s}\right)(\lambda-(j+1))(\lambda-(j+1))(\lambda-(j+2)) \cdots\left(\lambda-q^{k}\right)$. Let $M^{\prime}=M^{\prime \prime} / x$. By the standard recursion formula for the characteristic polynomial of a matroid we have

$$
P\left(M^{\prime} ; \lambda\right)=P(M ; \lambda)-P\left(M^{\prime \prime} ; \lambda\right)
$$

and therefore

$$
P\left(M^{\prime} ; \lambda\right)=(\lambda-1)(\lambda-q) \cdots\left(\lambda-q^{s}\right)(\lambda-(j+1))(\lambda-(j+2)) \cdots\left(\lambda-q^{k}\right) .
$$

Certainly $\overline{M^{\prime}}$ is a supersolvable rank $m-1$ tangential $k$-block over $G F(q)$ (the technique used to construct $M^{\prime}$ is precisely the technique used in the proof of Theorem 3.2). The characteristic polynomial of $M^{\prime}$ (and therefore $\overline{M^{\prime}}$ ) corresponds to the one given in the statement of the theorem for $r=m-1$ whenever $q^{s+1}-$ $(s+1) \geq q^{k}-(m-1)+2$ so assume that $q^{s+1}-(s+1)<q^{k}-m+3$. By the definition of $s, q^{s+1}-(s+1) \geq q^{k}-m+2$. Therefore $q^{s+1}-(s+1)=q^{k}-m+2$ and since $j=q^{k}-m+s+2$, we have $j+1=q^{s+1}$. Hence

$$
P\left(M^{\prime} ; \lambda\right)=(\lambda-1)(\lambda-q) \cdots\left(\lambda-q^{s}\right)\left(\lambda-q^{s+1}\right)(\lambda-(j+2)) \cdots\left(\lambda-q^{k}\right)
$$

and it is readily verified that this (with appropriate relabelling) is the characteristic polynomial given in the statement of the theorem for $r=m-1$ and $q^{s+1}-(s+1)=$ $q^{k}-m+2$.

As a straightforward illustration of the above theorem we give examples of characteristic polynomials of tangential 2-blocks over $G F(3)$.

EXAMPLE 3.6. Over $G F(3)$ there exist supersolvable tangential 2-blocks with the following ranks and characteristic polynomials.

Rank Characteristic polynomial

$\begin{array}{lll}9 & (\lambda-1)(\lambda-2) \cdots(\lambda-9) & M\left(K_{10}\right) \\ 8 & (\lambda-1)(\lambda-3)(\lambda-4) \cdots(\lambda-9) & T_{M\left(K_{3}\right)}\left(M\left(K_{10}\right)\right) \\ 7 & (\lambda-1)(\lambda-3)(\lambda-5)(\lambda-6) \cdots(\lambda-9) & \\ 6 & (\lambda-1)(\lambda-3)(\lambda-6)(\lambda-7)(\lambda-8)(\lambda-9) & \\ 5 & (\lambda-1)(\lambda-3)(\lambda-7)(\lambda-8)(\lambda-9) & \\ 4 & (\lambda-1)(\lambda-3)(\lambda-8)(\lambda-9) & P G(2,3) \\ 3 & (\lambda-1)(\lambda-3)(\lambda-9) & \end{array}$




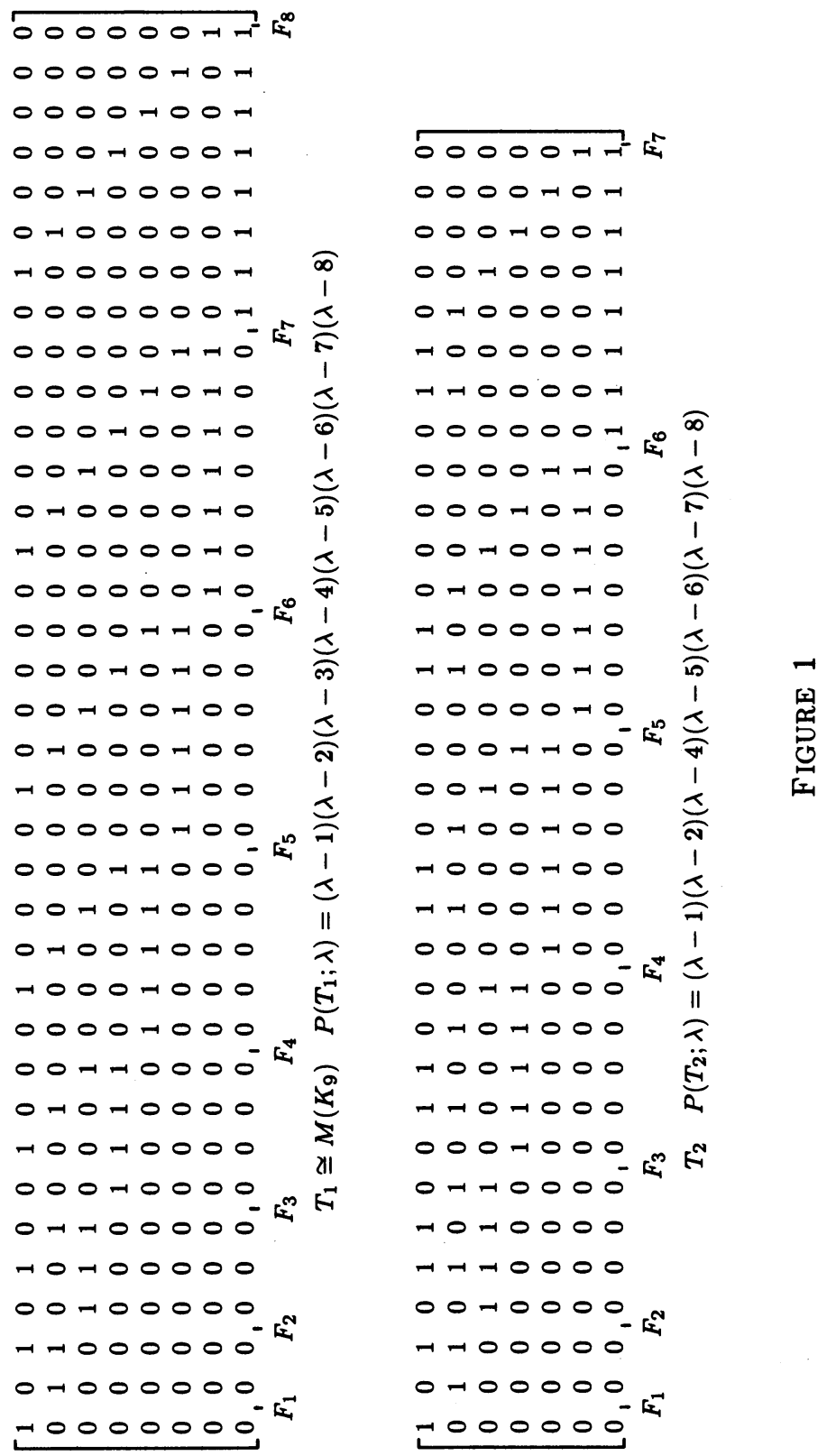




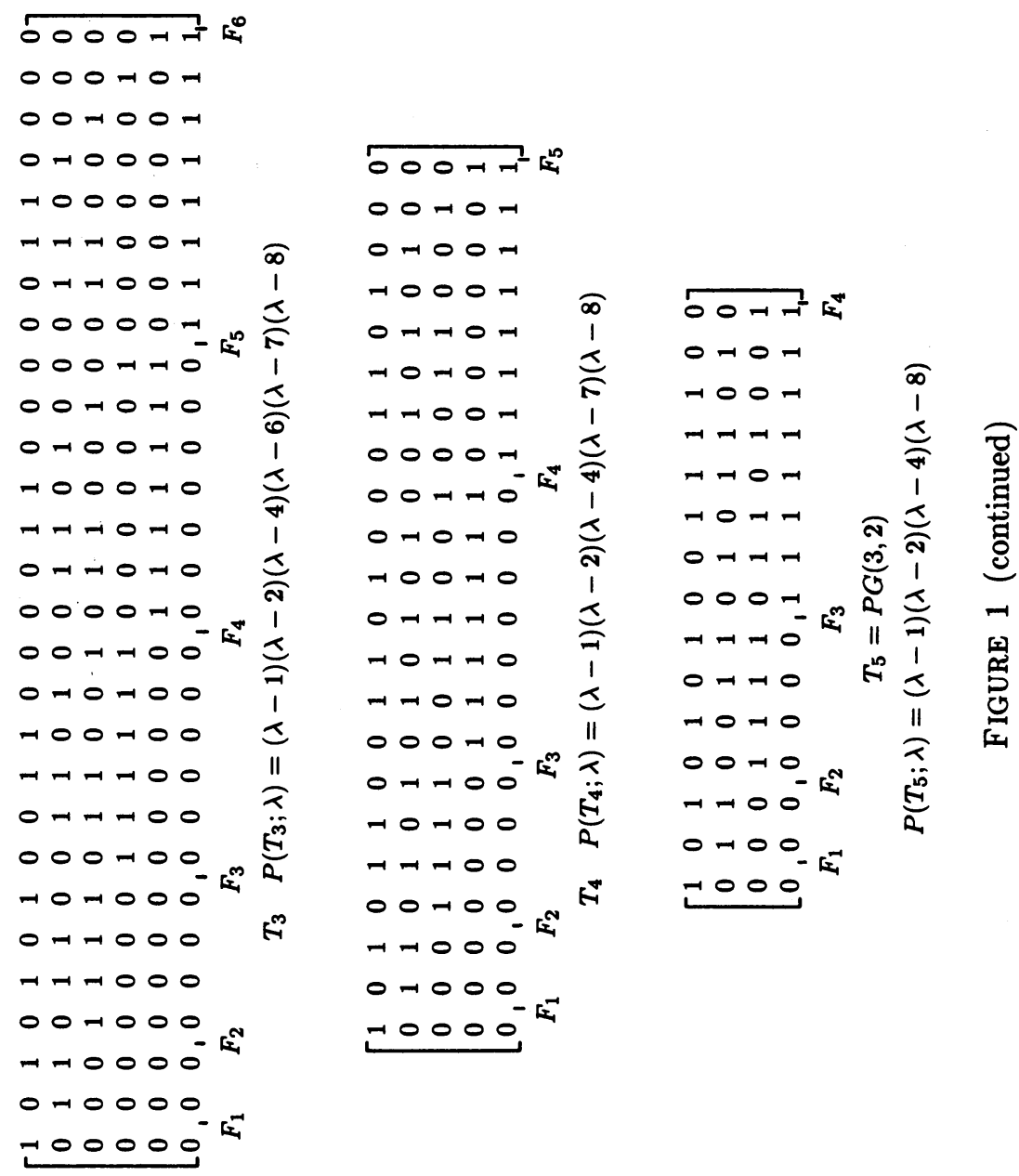


Note that, in general, for fixed values of $r$ and $q$ there may exist many nonisomorphic rank $r$ tangential $k$-blocks over $G F(q)$ whose characteristic polynomials are equal to that given in the statement of Theorem 3.5. There may also exist others with different characteristic polynomials.

EXAMPLE 3.7. Tangential 3-blocks over $G F(2)$. Figure 1 gives matrix representations of tangential 3 blocks over $G F(2) . T_{1}$ is $M\left(K_{9}\right)$ and $T_{5}$ is $P G(3,2)$. $T_{i+1}$ is obtained from $T_{i}$ by the method of Theorems 3.2 and 3.5. In particular $T_{2}$ is obtained from $T_{1}$ by extending by the vector $[1,1,1,0,0,0,0,0]^{T}$ and then contracting this vector from the extended matroid, $T_{3}$ is obtained from $T_{2}$ by adding and contracting $[1,1,1,1,0,0,0]^{T}, T_{4}$ is obtained from $T_{3}$ by adding and contracting $[1,0,1,1,0,0]^{T}$ and $T_{5}$ is obtained from $T_{4}$ by adding and contracting $[1,1,1,1,0]^{T}$. The point added to $T_{i}$ and subsequently contracted yields a matroid $T_{i}^{\prime}$ with $\overline{T_{i}^{\prime}}=T_{i+1}$. The columns have been ordered to correspond to a saturated chain of modular flats. In each case the flat $F_{i}$ consists of all columns to the left of the symbol " $F_{i}$ ". In most cases the choice of the point to be added and subsequently contracted is not unique. For example in going from $T_{2}$ to $T_{3}$ one could also have chosen $[1,0,1,1,0,0,0]^{T}$ or $[0,1,1,1,0,0,0]^{T}$. It is routine to verify that both alternative choices yield $T_{3}$. In fact, starting from $M\left(K_{9}\right)$ and using the method of Theorems 3.2 and 3.5 it seems that one always obtains tangential $k$-blocks isomorphic to $T_{2}, T_{3}$ and $T_{4}$ although I have not checked this thoroughly. Note that $T_{2}, T_{3}, T_{4}$ and $T_{5}$ all have a minor isomorphic to $P G(2,2)$ and are therefore not graphic.

\section{REFERENCES}

1. T. H. Brylawski, Modular constructions for combinatorial geometries, Trans. Amer. Math. Soc. 203 (1975), 1-44.

2. T. H. Brylawski and J. G. Oxley, Several identities for the characteristic polynomial of a combinatorial goemetry, Discrete Math. 31 (1980), 161-170.

3. H. H. Crapo and G.-C. Rota, On the foundations of combinatorial theory: Combinatorial geometries, M.I.T. Press, Cambridge, Mass., 1970.

4. R. Stanley, Modular elements of geometric lattices, Algebra Universalis 1 (1971), 214-217.

5. _ Supersolvable lattices, Algebra Universalis 2 (1972), 197-217.

6. D. J. A. Welsh, Matroid theory, London Math. Soc. Monographs, No. 8, Academic Press, New York, 1976.

7. __ Colouring problems and matroids, Surveys in Combinatorics, London Math. Soc. Lecture Notes, Vol. 38 (B. Bollobas, Ed.), Cambridge Univ. Press, 1979.

8. __ Colourings, flows and projective geometry, Niew Arch. Wisk. (3) 28 (1980), 159-176.

9. G. P. Whittle, Modularity in tangential k-blocks, J. Combin. Theory B 42 (1987), 24-35.

10. _ Some aspects of the critical problem for matroids, Ph.D. Thesis, Univ. of Tasmania, 1985.

Department of Mathematics, University of TASMANia, G.P.O. Box 252C, hobart, Tasmania 7001, Australia 